\title{
Editorial
}

\section{Developments in interventional catheterisation and progress in surgery for congenital heart disease: achieving a balance}

Progress in the understanding and treatment of congenital heart disease has been one of the most important medical achievements of the last 15 years. Many factors have been responsible including the advent of cross sectional and Doppler echocardiography together with developments in interventional catheterisation. However, the improved outcome for these children must largely be ascribed to the emergence of cardiac surgeons with a major interest in paediatric cardiac surgery and the development of the team approach which is now fundamental to the current philosophy of management.

Part of the future challenge lies in reconciling the improvements in outcome from cardiac surgery with the cardiologist's drive to develop interventional techniques. Balloon dilatation has become the treatment of choice for certain congenital heart lesions including pulmonary valve stenosis ${ }^{1}$ and aortic re-coarctation. ${ }^{2}$ In other conditions, for example aortic valve stenosis, ${ }^{3}$ peripheral pulmonary artery stenosis, ${ }^{4}$ and the embolisation of aortopulmonary collaterals, ${ }^{5}$ the role of catheter techniques is less well established but appears promising in the short and intermediate term. However, the stage is now being reached in the United Kingdom when more complicated interventional techniques are being applied to complex forms of congenital heart disease on an experimental basis. In this edition of the British Heart fournal there are two papers that describe new catheter techniques and it is the purpose of this editorial to attempt to place this work into the context of present knowledge and conventional treatment.

\section{Laser assisted valve dilatation}

Rosenthal et al (page 556) describe laser assisted valve dilatation for valvar atresia in 11 patients. Eight of the patients had pulmonary atresia with intact ventricular septum and two had pulmonary atresia with a ventricular septal defect. Though the numbers are small, this group has the largest experience in the world and the technical details are clearly presented. These workers have not clarified their criteria for patient selection, however. Previous surgical experience has confirmed that the management of an individual patient must depend almost entirely on the associated structural abnormalities remote from the atretic valve. Successful treatment therefore requires detailed prior assessment of the individual anatomy to decide whether a valvotomy, surgical or otherwise, is likely to be beneficial. Patients with pulmonary atresia and ventricular septal defect frequently have inherent abnormalities of the pulmonary arterial system and lobar anatomy which dictate management protocols. ${ }^{6}$ In the minority of children with normal central pulmonary arteries and without arborisation abnormalities surgical correction should be considered and can be achieved with low morbidity and mortality. One of the patients in this paper with pulmonary atresia and ventricular septal defect appears suitable for surgical correction and the indications for undertaking laser treatment at this age (2 years) needs further clarification.
In those with pulmonary atresia and an intact ventricular septum the management has to be based on the size and morphology of the right ventricle and the presence or absence of right ventricular sinusoids. ${ }^{7-9}$ The ratio of the tricuspid valve annulus to the mitral valve annulus is central to management strategy. ${ }^{10}$ In patients with pulmonary atresia and intact ventricular septum with a diminutive right ventricle, particularly those with sinusoids where ischaemia may contribute to a poor outcome, ${ }^{11}$ the establishment of right ventricle to pulmonary artery continuity may not be helpful for several reasons. Firstly, these ventricles are severely restrictive and do not grow, so that the angiographic demonstration of forward flow does not mean there will be sufficient pulmonary blood flow to allow adequate oxygenation.

Furthermore, decompression of a right ventricle with significant sinusoids by any method may be deleterious as part or all of their coronary circulation may be right ventricular dependent. ${ }^{12}$ This paper demonstrates that it was possible to cross the pulmonary valve in two patients with sinusoids but the rationale for doing so is not clear. Even those patients with a larger right ventricle may not derive immediate benefit from a valvotomy because of reduced compliance. This will clearly be the case whether the valvotomy is achieved surgically or by the laser technique. Ensuring a secure alternative (if temporary) pulmonary blood flow is frequently a priority and this problem is highlighted in Rosenthal et al paper. Balloon dilatation of the ductus arteriosus was performed as an alternative to shunting in five of their patients (including one who required duct dilatation twice). This technique to maintain ductal patency remains controversial and is at best temporary. ${ }^{13}$ The use of a standard modified Blalock-Taussig shunt, a straightforward non-bypass surgical procedure with proven low morbidity and mortality, ${ }^{914}$ would have avoided the need to use a second experimental procedure in the management of these patients.

Recent publications of the results of conventional surgery have not been cited, but where surgical management is based on detailed anatomical considerations, short and intermediate term survival rates in excess of $80 \%$ have been reported in patients with pulmonary atresia and an intact ventricular septum. ${ }^{7-9}$ The stated objective of Rosenthal et al paper to assess the "efficacy and safety" of the laser technique has therefore not been achieved: three patients with pulmonary atresia and intact ventricular septum developed tamponade and two of these died. There was an overall mortality at 11 months follow up of $50 \%$ (four deaths out of eight patients).

\section{Stenting and banding}

Gibbs et al (page 551) describe a novel approach for the treatment of the hypoplastic left heart syndrome, combining interventional catheterisation with surgery. Their results have to be compared with established protocols before the place of their treatment in the management of 
this depressing condition can be assessed. The aim of any treatment has to be to prepare the child for a Fontan circulation or to palliate to an age when cardiac transplantation becomes a feasible option. Norwood ${ }^{15}$ has evolved a three stage approach though few centres have been able to reproduce his results. ${ }^{16}$ The first stage involves reconstruction of the entire hypoplastic aortic arch. The main pulmonary artery is detached from the branches and forms the proximal aorta while a modified BlalockTaussig shunt controls pulmonary blood flow and an atrial septectomy decompresses the left atrium. The right ventricle becomes the systemic chamber. A bi-directional cavopulmonary shunt is electively undertaken at approximately 6 months and the third stage involves completion of the Fontan circuit. From January 1989, 151 patients have entered this program with 42 early and five late deaths. Seventy eight of the 104 survivors have undergone the second stage with nine deaths and a further 27 have undergone completion of the Fontan without mortality. Clearly any new treatment that potentially simplifies management and enables these results to be reproduced elsewhere will be welcome.

Gibbs et al technique aims to allow the systemic circulation to be maintained through the stented ductus arteriosus while protecting the pulmonary vasculature with separate branch pulmonary artery bands placed surgically. Unfortunately, there are several ways that this approach will complicate future management. Firstly, -ductal stenting alone cannot obviate the need for radical aortic arch reconstruction. The aortic arch is almost universally hypoplastic as part of the syndrome, and coronary and cerebral blood flow must ultimately become severely compromised, though this issue is not raised by Gibbs et al. Secondly, an $8 \mathrm{~mm}$ ductus (the final stent diameter) will become inherently obstructive as the child grows. The assertion in the paper that future dilatation of the stent might be possible is speculative, and the hope of a future Fontan circulation appears distant in the presence of obstruction to ventricular outflow. Thirdly, adequate protection of the pulmonary vasculature by banding is critical to the success of a future Fontan procedure. However, it is known from the experience with persistent truncus arteriosus that banding of the branch pulmonary arteries is very unsatisfactory. ${ }^{17}$ Even if adequate protection of the lungs can be achieved, reconstruction of the distorted branch pulmonary arteries will be necessary and is known to be an independant risk factor for the success of both transplantation ${ }^{18}$ and the Fontan procedure. ${ }^{19}$ In the paper by Gibbs et al ductal stenting and banding of the branch pulmonary arteries resulted in a $50 \%$ immediate mortality (two out of four patients) and one patient remains in pulmonary oedema secondary to increased pulmonary blood flow. The high early mortality and complicated future management resulting from this approach mean that its place in the treatment of hypoplastic left heart syndrome is uncertain.

While interventional cardiac catheterisation continues to contribute significantly to the management of congenital heart disease, it is important that the avoidance of surgery in itself is not the main measure of success. These procedures should result in a comparable or reduced morbidity and mortality and should not complicate future management. When novel palliative interventional procedures are being developed a clear long-term plan must be made which offers realistic hopes for a satis- $I$ factory quality of life as well as survival for these younge children. Close cooperation between surgeons and physicians will continue to be central to the development of management protocols.

Wessex Cardiothoracic Centre,

Southampton General Hospital

A P SALMONO B R KEETON

B SETHIAฉ̆

Heart Unit,

Birmingham Children's Hospital

1 Tynan M, Baker EJ, Rohmer J, Jones ODH, Reidy JF, Joseph MC, Ottenkamp J. Percutaneous balloon pulmonary valvuloplasty. Br Heart f 1985;53:520-4.

2 Hellenbrand WE, Allen HD, Golinko RJ, Hagler DJ, Lutin W, Kan Jö Balloon angioplasty for aortic re-coarctation: Results of the valvuloplasty and angioplasty of congenital anomolies registry. $\mathrm{Am} \mathrm{f}$ Cardio? 1990;65:793-7.

3 Keane JF, Perry SB, Lock JE. Balloon dilatation of congenital valvularo aortic stenosis. $\mathcal{F}$ Am Coll Cardiol 1990;16:457-8.

4 Kan JS, Marvin WJ, Bass JL, Muster A, Murphy J. Balloon angioplasty branch pulmonary artery stenosis: results of the Valvuloplasty andAngioplasty of Congenital Anomolies Registry. Am $\mathcal{F}$ Cardiol $1990 €$ 65:798-801.

5 Perry SB, Radtke W, Fellows KE, Keane JF, Lock JE. Coil embolisationd of aortopulmonary collateral vessels and shunts in patients with congen of aortopulmonary collateral vessels and shunts in pat
ital heart disease. $₹ \mathrm{Am} \mathrm{Coll} \mathrm{Cardiol} \mathrm{1989;13:100-8.}$

6 McCartney FJ, Scott O, Deverall PB. Haemodynamic and anatomicate characteristics of pulmonary blood supply in pulmonary atresia with ventricular septal defect-including a case of persistent fifth aortic arch? Br Heart f 1974;36:1049-60.

7 Joshi SV, Brawn WJ, Mee RBB. Pulmonary atresia with intact ventriculas septum. F Thorac Cardiovasc Surg 1986;91:192-9.

8 Hawkins JA, Thorne JK, Boucek MM, Orsmond GS, Ruttenberg HD Veasy LG, McGough EC. Early and late results in pulmonary atresiक and intact ventricular septum. I Thorac Cardiovasc Surg 1990;100 492-7.

9 Vosa C, Arciprete P, Caianiello G, Palma G. Pulmonary atresia witto $\overrightarrow{\vec{D}}$ intact ventricular septum: Is it possible to improve survival? Cardio $\bar{B}$ Young 1992;2:391-4.

10 Amodeo A, Keeton BR, Sutherland GR, Monro JL. Pulmonary atresig with intact ventricular septum: is neonatal repair advisable? Eur Cardiothorac Surg 1991;5:17-21.

11 Freedom RM, Benson LM, Trussler GA. Pulmonary atresia and intact ventricular septum: A consideration of the coronary circulation and ven $\frac{0}{7}$ triculo-coronary artery connexions. Ann Cardiac Surg 1989;38:38-44.

12 Ueda K, Saito A, Nakano H, Hamazaki Y. Absence of proximal coronary arteries associated with pulmonary atresia. Am Heart $f 1983 ; 106: 596-8,-$. Walsh KP, Sreeram N, Franks R, Arnold R. Balloon dilatation of the arterial duct in congenital heart disease. Lancet 1992;339:331-2.

14 Fermanis GG, Ekangaki AK, Salmon AP, Keeton BR, Shore DF, Lamb RK, Monro JL. Twelve year experience with the modified BlalockO Taussig shunt in neonates. Eur $\mathcal{F}$ Cardiothorac Surg 1992;6:586-9.

15 Norwood, WI, Jnr. Hypoplastic left heart syndrome. Ann Thorac Surg 1991;52:688-95.

16 Meliones JN, Snider AR, Bove EL, Rosenthal A, Rosen DA. LongitudinaFresults after first stage palliation for hypoplastic left heart syndromeN
Circulation 1990;82 (5 suppl IV):151-6.

17 Pearl JM, Laks H, Drinkwater DC, Milgalter E, Orrin-Ailloni-Charas N Giacobetti F, George B, Williams R. Repair of truncus arteriosus in infancy. Ann Thorac Surg 1991;52:780-6.

18 Trento A, Griffith BP, Fricker FJ, Kormos RL, Armitage J, Hardesty RI Lessons learned in paediatric heart transplantation. Ann Thorac Surr 1989;48:617-23.

19 Fontan F, Kirklin JW, Fernandez G, Costa F, Naftel DC, Tritto F Blackstone EH. Outcome after a "perfect" Fontan operation. Circulations 1990;81:1520-36. 